\title{
BMJ Open Measuring engagement in advance care planning: a cross-sectional multicentre feasibility study
}

\author{
Michelle Howard, ${ }^{1}$ Aaron J Bonham, ${ }^{2}$ Daren K Heyland, ${ }^{3,4}$ Rebecca Sudore, ${ }^{5,6}$ \\ Konrad Fassbender, ${ }^{7}$ Carole A Robinson, ${ }^{8}$ Michael McKenzie, ${ }^{9}$ Dawn Elston, ${ }^{1}$ \\ John $\mathrm{J}$ You $^{2,10}$
}

To cite: Howard M, Bonham AJ, Heyland DK, et al. Measuring engagement in advance care planning: a cross-sectional multicentre feasibility study. BMJ Open 2016;6: 010375 .

doi:10.1136/bmjopen-2015010375

- Prepublication history for this paper is available online. To view these files please visit the journal online (http://dx.doi.org/10.1136/ bmjopen-2015-010375).

Received 26 October 2015 Revised 20 April 2016 Accepted 10 May 2016

CrossMark

For numbered affiliations see end of article.

Correspondence to Dr Michelle Howard; mhoward@mcmaster.ca

\section{ABSTRACT}

Objectives: To assess the feasibility, acceptability and clinical sensibility of a novel survey, the advance care planning (ACP) Engagement Survey, in various healthcare settings.

Setting: A target sample of 50 patients from each of primary care, hospital, cancer care and dialysis care settings.

Participants: A convenience sample of patients without cognitive impairment who could speak and read English was recruited. Patients 50 and older were eligible in primary care; patients 80 and older or 55 and older with clinical markers of advanced chronic disease were recruited in hospital; patients aged 19 and older were recruited in cancer and renal dialysis centres.

Outcomes: We assessed feasibility, acceptability and clinical sensibility of the ACP Engagement Survey using a 6-point scale. The ACP Engagement Survey measures ACP processes (knowledge, contemplation, self-efficacy and readiness) on 5-point Likert scales and actions (yes/no).

Results: 196 patients (38-96 years old, 50.5\% women) participated. Mean $( \pm S D)$ time to administer was $48.8 \pm 19.6$ min. Mean acceptability scores ranged from $3.2 \pm 1.3$ in hospital to $4.7 \pm 0.9$ in primary care, and mean relevance ranged from $3.5 \pm 1.0$ in hospital to $4.9 \pm 0.9$ in dialysis centres ( $p<0.001$ for both). The mean process score was $3.1 \pm 0.6$ and the mean action score was $11.2 \pm 5.6$ (of a possible 25).

Conclusions: The ACP Engagement Survey demonstrated feasibility and acceptability in outpatient settings but was less feasible and acceptable among hospitalised patients due to length. A shorter version may improve feasibility. Engagement in ACP was low to moderate.

\section{INTRODUCTION}

Advance care planning (ACP) is a communication process where people plan for a time when they cannot make decisions for themselves. It includes reflection, deliberation and determination of a person's values and

\section{Strengths and limitations of this study}

- The study was conducted across a wide range of healthcare settings.

- The study used a measure of feasibility, acceptability and clinical sensibility to assess a comprehensive survey of advance care planning behaviours.

- Convenience sample of patients and inclusion of only those who could speak and understand English, who were not cognitively impaired and could hear well enough to participate in the interview may limit the generalisability of findings.

- Cultural factors could not be evaluated because the majority of patients reported their ethnicity as Caucasian/white.

wishes and, if appropriate, preferences for treatments at the end of life. It also includes communication between an individual and his or her loved ones, future substitute decision maker(s) (SDM) and healthcare provider(s) about these values and wishes. ${ }^{1}$

ACP can improve the end-of-life experiences of patients and families. ${ }^{2}{ }^{3}$ In a randomised trial, end-of-life wishes were more than twice as likely to be known and followed for individuals receiving an ACP intervention compared to controls. In addition, family members of patients who died but who had received the ACP intervention reported significantly less stress, anxiety and depression than those in the control group. ${ }^{3}$ Despite the known benefits of ACP, people with lifelimiting illnesses facing an acute health crisis have often not adequately conveyed their preferences. ${ }^{4}$ In a Canadian survey, it was found that although approximately half of the respondents had engaged in some type of end-of-life discussion with family or friends, communication with healthcare providers about such discussions was much less 
common and only $9 \%$ of respondents had discussed their preferences for future medical care with a healthcare provider. $^{5}$

The reported outcome of interventions to improve ACP has often been the presence of a written advance directive document. ${ }^{6}{ }^{7}$ The advance directive typically specifies a SDM and states a person's healthcare decisions around resuscitation or other treatments. The limitations of advance directives or living wills are that they have little utility for predicting wishes for care in an actual clinical setting, ${ }^{8}$ have no legal standing in some jurisdictions and are often not available at the time healthcare decisions need to be made. In addition, the presence of an advance directive does not guarantee that a patient has reflected on a variety of health states that may be experienced in relation to his or her desired quality of life or that the patient has communicated his or her values and preferences to the SDM. For ACP to be most effective, there are multiple behaviours to consider, including identifying a SDM, identifying one's values, communicating verbally or in writing the values and preferences to the SDM, communicating with other family or friends who may influence decisions and communicating with healthcare providers. ${ }^{9}$ Thus, measures that include only completion of advance directives do not reflect whether the intervention addressed the full range of ACP-related behaviours, and interventions may be deemed unsuccessful even though they may be effective in moving people along the behaviour change pathway to actions.

To measure the full range of engagement in ACP behaviours and to develop and evaluate the effectiveness of tools to facilitate ACP beyond advance directive documentation, we need a feasible and valid self-report measure of ACP behaviours. ACP engagement, in this article, is the term used to describe a person's behaviour with respect to the various aspects of ACP according to a stage of behaviour change (knowledge, contemplation, self-efficacy and readiness).

The ultimate goal of ACP is to improve concordance between a patient's wishes and actual healthcare received, but measurement of this outcome is fraught with challenges including that it is a very 'distal' outcome and so can be subject to many other influences besides an initial 'upstream' ACP intervention. Thus, a comprehensive measure is needed to capture in a more 'proximal' fashion whether ACP is actually occurring (beyond completion of advance directives) in response to an ACP intervention. Based on Social Cognitive Theory and a stage-of-change conceptual model, the ACP Engagement Survey has been developed and its reliability and validity examined among older adults in community and ambulatory care settings in the USA. ${ }^{9}$ The ACP Engagement Survey is intended to measure the impact of interventions, by detecting changes in behaviour along the spectrum. It allows measurement at a more granular level to provide information on where specifically the intervention is having an impact or not, and how much of an impact. To date, the ACP Engagement Survey has not been tested for feasibility of use among varying age groups, disease processes and various healthcare settings.

The objective of this study was to evaluate the feasibility, acceptability and clinical sensibility of using the ACP Engagement Survey in primary care, hospital care, cancer care and dialysis care.

\section{MATERIALS AND METHODS}

From April to September 2014, we conducted a crosssectional study by administering the ACP Engagement Survey $^{9}$ to a convenience sample of patients in primary care, (inpatient) hospital, (outpatient) cancer care and (outpatient) dialysis care (outpatient) in five different Canadian cities in the provinces of British Columbia, Alberta and Ontario.

\section{Recruitment and eligibility}

A convenience sample of patients who did not have cognitive impairment and who could speak and read English were recruited from four primary care practices (aged 50 and older), two hospitals (aged 80 and older, or 55 and older with clinical markers of advanced chronic disease), four cancer centres (aged 19 and older) and one renal dialysis centre (aged 19 and older). A sample size of 50 patients from each of hospital, primary care, cancer care and dialysis care was targeted.

\section{Feasibility assessment}

Using an instrument from a previous study assessing feasibility of a patient survey about end-of-life communication and decision-making, ${ }^{10}$ we assessed the feasibility of the ACP Engagement Survey by asking the participants (1) to rate the language, clarity, comprehensiveness, acceptability, relevance and fit of response options on a 6-point Likert scale (1-very poor, 2-poor, 3-fair, 4-good, 5-very good and 6-excellent) and (2) to rate emotional burden on a 5-point Likert scale (1-not at all upsetting, 2-a little upsetting, 3-somewhat upsetting, 4-fairly upsetting and 5-extremely upsetting), which was reverse coded at the analysis stage. We also recorded the time taken to administer the survey.

\section{ACP Engagement Survey}

The ACP Engagement Survey (hereafter referred to as 'the Survey') measures multiple ACP behaviours. ${ }^{9}$ It is based on a conceptual framework of ACP articulating four behaviours that relate to (1) engaging decision makers, (2) considering acceptable quality of life, (3) considering flexibility for decision makers and (4) asking doctors questions. Degree of engagement with each ACP behaviour is gauged through questions based on Social Cognitive Theory and Behaviour Change Theory. Within each domain, questions pertain to processes, including knowledge, contemplation, self-efficacy 
and readiness, and actions such as having asked someone to be an SDM. Overall process and action scores can be measured separately. The original Survey contained 49 items (31 process measures and 18 action measures). The Survey was created to ensure appropriate language for older adults and all literacy levels and has been pilot tested in the USA in adults aged 55 and older in acute care, outpatient clinics, nursing home and community health clinics. Overall internal consistency (Cronbach's $\alpha$ ) for the ACP process measures was 0.94. Internal consistency remains high within each subscales ranging between 0.84 and 0.94 . Overall 1-week test-retest reliability (intraclass correlations) for the process measures was 0.70 (subscales between 0.56 and 0.70 ) and for action measures was 0.87 (subscales between 0.57 and 0.87$).^{9}$

Subsequent to the published validated Survey, 33 additional questions, which were not validated, were added in preparation for additional ACP studies including the current study. The new items follow the behaviour change construct structure of the original Survey. The need for new items arose from further consultation with patients and key experts in ACP, including some of the current study's authors. The earlier version was felt to be lacking in two aspects: questions about medical care desired if one were very ill or near the end of life were felt by key experts to be needed within the quality-of-life domain, and questions about other family and friend involvement were added in response to previous research about the role of family decision-making in some cultures ${ }^{11}$ and direct input from patients (RS, personal communication, unpublished data, July 2015). Eighteen questions (13 process and 5 action items) were added to the quality-of-life domain and 12 questions (9 process and 3 action items) were added about involvement of other family and friends. Furthermore, two process questions about readiness to decide on a medical decision maker and about health situations that would make life not worth living, one process question about talking with the doctor about flexibility and one process question about self-efficacy talking with the doctor about flexibility were added for symmetry of the behaviour change constructs within the Survey. One action item about having decided on questions to ask the doctor was deleted due to participant feedback indicating confusion about the question. The subsequent survey contained 82 scored items (57 process items and 25 action items). The remaining items in the 116-item version used in the current study were informational questions (eg, If you became too sick to make your own decisions, can you think of anyone in your life right now, such as family or friends, who may be able to help make medical decisions for you?) and questions on satisfaction with discussions with medical decision maker, family and friends and doctor.

While the role of family members/surrogates is key in ACP, the Survey was designed to be answered by an individual and does not attempt to assess surrogates' perspectives. However, the Survey does include domains relating to the respondents' engagement of and communication with surrogates and other family or friends.

\section{Survey administration}

The Survey was administered face to face by trained interviewers. We trained interviewers using a demonstration video that was created by the Survey developer. In addition to the Survey items, demographic data were also obtained including age, gender, race, education and marital status. We also asked about the importance of religion or spirituality, self-reported health status and quality of life.

\section{Ethics}

Ethics approval for the study was obtained from the Research Ethics Board of each participating institution (Hamilton Integrated Research Ethics Board, British Columbia Cancer Agency Research Ethics Board, Queen's University Health Sciences and Affiliated Teaching Hospitals Research Ethics Board, and the Health Research Ethics Board-Health Panel and Health Research Ethics Board of Alberta-Cancer Committee). Informed consent for participating in the study was obtained verbally (as approved by each research ethics board) in all settings except one acute care setting in Hamilton where written consent was also obtained.

\section{Statistical analyses}

For feasibility (0-point-6-point scale, emotional burden 0 -point-5-point scale) and time to complete the survey, we computed means and SDs. For the Survey analyses, we calculated average 5-point Likert scores for ACP processes as described previously. ${ }^{9}$ All response options in the Survey are worded such that a score of 1 indicates the lowest level of engagement and 5 indicates the highest. Scores were computed for processes overall and for each individual domain (knowledge, contemplation, self-efficacy and readiness). Overall action scores were calculated as the sum of 'yes' responses across all items (0-25) and for each individual domain: decision maker (score: 0-5), quality of life (score: 0-10), flexibility (score: $0-5$ ) and ask doctor questions (score: 0-5). If a patient had a missing response for a question within a process score, the scale mean was computed ignoring the missing data. If a response to a question was missing within an action scale, it was assumed that the action was not carried out.

To describe the samples, we presented demographic characteristics and self-reported health variables by healthcare setting. We compared feasibility across the four different healthcare settings using the Kruskal-Wallis non-parametric test. Where a statistically significant difference was found between settings, pairwise post hoc tests were performed using the Bonferroni correction for multiple comparisons (two-sided). The criterion of statistical significance was set at $\alpha=0.05$. 
We also summarised Survey scores for the overall study sample and descriptively examined the scores on the Survey by healthcare setting.

Statistical analyses were conducted using IBM SPSS V.22.0.

\section{RESULTS}

One hundred and ninety-six patients completed the survey. The number of patients ranged from 20 in the dialysis care setting to 63 in the primary care settings. The mean age of patients was 66.3 years in cancer settings, 69.0 years in the dialysis care setting, 71.0 years in primary care settings and 81.7 years in the hospital settings (table 1).

Approximately half of the patients were women. Half of the patients $(49.2 \%)$ in hospital settings reported their health as good, very good or excellent, increasing to $82.0 \%$ in primary care settings. The majority of patients reported being Caucasian/white $(91.4 \%)$, with a slightly lower proportion being Caucasian/white in the cancer settings $(85.7 \%)$.
Feasibility, acceptability, clinical sensibility and burden

There was a statistically significant difference between settings for all the feasibility and acceptability items. Mean scores for acceptability and feasibility items were $>4$ on 6-point scales for all items in all settings except hospital where scores were lower (table 2).

In the pairwise comparisons, for all items, hospitalised patients were significantly different from patients in each of the other settings, and patients in primary care, cancer care and dialysis care did not differ significantly from each other on any of the items. Overall, there was low emotional burden; mean scores were 4 or higher in all settings (score of 5 being 'not at all upsetting').

The mean length of interview was $48.8 \pm 19.6 \mathrm{~min}$ and there was a statistically significant difference in length of interview between settings. Interviews with patients in the hospital setting $(59.3 \pm 21.9 \mathrm{~min})$ were significantly longer than interviews with patients in the cancer care settings $(46.7 \pm 14.5 \mathrm{~min})$, dialysis settings (mean: 33.8 $\pm 7.6 \mathrm{~min}$ ) and primary care settings (mean: 43.3 $\pm 17.4 \mathrm{~min}$ ). Dialysis care patients also had significantly

Table 1 Demographic characteristics of the study participants in the different healthcare settings

\begin{tabular}{|c|c|c|c|c|c|}
\hline & Total $(\mathrm{N}=196)$ & Cancer $(n=49)$ & Hospital $(n=64)$ & Dialysis $(n=20)$ & Primary care $(n=63)$ \\
\hline \multicolumn{6}{|l|}{ Age } \\
\hline Mean (SD) & $72.7(12.4)$ & $66.3(11.4)$ & $81.7(10.0)$ & $69.0(12.5)$ & $69.4(10.2)$ \\
\hline Median (1st, 3rd quartile) & $74(63,83)$ & $64(57,75.5)$ & $84(80,89.8)$ & $67.5(60.3,80.5)$ & $71.0(60.8,78)$ \\
\hline Range & $38-96$ & $38-86$ & $55-96$ & $43-87$ & $50-90$ \\
\hline$<65$ years & $55(28.4)$ & 23 (47.9) & $6(9.4)$ & $7(35.0)$ & $17(27.4)$ \\
\hline$\geq 65$ years & 139 (71.6) & $25(52.1)$ & $58(90.6)$ & $13(65.0)$ & $45(72.6)$ \\
\hline \% Women & 99 (50.5) & $16(32.7)$ & $35(54.7)$ & $12(60.0)$ & $36(57.1)$ \\
\hline \multicolumn{6}{|l|}{ Race/ethnicity } \\
\hline White & $180(92.8)$ & $42(85.7)$ & $62(98.4)$ & $19(95.0)$ & 57 (91.9) \\
\hline Other & $14(7.2)$ & 7 (14.3) & $1(1.6)$ & $1(5.0)$ & $5(8.1)$ \\
\hline $\begin{array}{l}\text { Education, } \leq \text { high school } \\
\text { Health status }\end{array}$ & $101(52.3)$ & $16(33.3)$ & $44(69.8)$ & $10(50.0)$ & $31(50.0)$ \\
\hline Good/very good/excellent & $124(64.6)$ & $30(62.5)$ & $31(49.2)$ & $13(65.0)$ & $50(82.0)$ \\
\hline Fair/poor & 68 (35.4) & $18(37.5)$ & $32(50.8)$ & $7(35.0)$ & $11(18.0)$ \\
\hline \multicolumn{6}{|l|}{ Quality of life } \\
\hline Good/very good/excellent & $147(76.2)$ & $36(75.0)$ & $41(65.1)$ & $13(65.0)$ & 57 (91.9) \\
\hline Fair/poor & $46(23.8)$ & $12(25.0)$ & $22(34.9)$ & $7(35.0)$ & $5(8.1)$ \\
\hline \multicolumn{6}{|l|}{ Married/long-term relationship } \\
\hline No & 79 (40.9) & $13(27.1)$ & $38(60.3)$ & $11(55.0)$ & $17(27.4)$ \\
\hline Yes & $114(59.1)$ & 35 (72.9) & $25(39.7)$ & $9(45.0)$ & $45(72.6)$ \\
\hline \multicolumn{6}{|c|}{ How important is spirituality/religion } \\
\hline Extremely important & $24(12.5)$ & $7(14.9)$ & $8(12.7)$ & $4(20.0)$ & $5(8.1)$ \\
\hline Very important & $46(24.0)$ & $7(14.9)$ & $13(20.6)$ & $6(30.0)$ & $20(32.3)$ \\
\hline Somewhat important & $66(34.4)$ & $16(34.0)$ & $25(39.7)$ & $3(15.0)$ & $22(35.5)$ \\
\hline Not very important & $29(15.1)$ & 7 (14.9) & $12(19.0)$ & $4(20.0)$ & $6(9.7)$ \\
\hline Not at all important & $25(13.0)$ & $8(17.0)$ & $5(7.9)$ & $3(15.0)$ & $9(14.5)$ \\
\hline Missing & $2(1.0)$ & $2(4.3)$ & $0(0.0)$ & $0(0.0)$ & $0(0.0)$ \\
\hline \multicolumn{6}{|c|}{ Formal religious group or practice } \\
\hline Protestant & $82(42.5)$ & $13(27.1)$ & $35(55.6)$ & $11(55.0)$ & $23(37.1)$ \\
\hline Catholic & 39 (20.2) & $8(16.7)$ & $19(30.2)$ & $2(10.0)$ & $10(16.1)$ \\
\hline Jewish & $1(0.5)$ & $0(0.0)$ & $1(1.6)$ & $0(0.0)$ & $0(0.0)$ \\
\hline Other & $13(6.7)$ & $2(4.2)$ & $0(0.0)$ & $7(35.0)$ & $21(33.9)$ \\
\hline None & $58(30.1)$ & $25(52.1)$ & $5(7.9)$ & $0(0.0)$ & 8 (12.9) \\
\hline
\end{tabular}


Table 2 Mean scores on feasibility items assessing the ACP Engagement Survey, by healthcare setting

\begin{tabular}{|c|c|c|c|c|c|}
\hline \multirow[b]{2}{*}{ Construct } & \multicolumn{5}{|c|}{ Mean (SD)* } \\
\hline & $\begin{array}{l}\text { Total } \\
(\mathrm{N}=177)\end{array}$ & $\begin{array}{l}\text { Cancer } \\
(n=47)\end{array}$ & $\begin{array}{l}\text { Hospital } \\
(n=50)\end{array}$ & $\begin{array}{l}\text { Dialysis } \\
(n=20)\end{array}$ & $\begin{array}{l}\text { Primary care } \\
(n=60)\end{array}$ \\
\hline Language/understanding of items $†$ & $4.3(1.0)$ & $4.5(1.1)$ & $3.7(1.0)$ & $4.5(0.9)$ & $4.6(0.9)$ \\
\hline Clarity† & $4.2(1.1)$ & $4.5(1.1)$ & $3.4(1.0)$ & $4.3(0.9)$ & $4.5(1.0)$ \\
\hline Comprehensiveness† & $4.3(1.1)$ & $4.5(1.0)$ & $3.5(1.0)$ & $4.6(0.8)$ & $4.7(1.0)$ \\
\hline Fit† & $3.9(1.2)$ & $4.1(1.0)$ & $3.2(1.3)$ & $4.4(0.9)$ & $4.2(1.0)$ \\
\hline Acceptability $†$ & $4.2(1.1)$ & $4.2(1.1)$ & $3.6(1.1)$ & $4.5(0.9)$ & $4.7(0.9)$ \\
\hline Relevance† & $4.2(1.1)$ & $4.4(1.0)$ & $3.5(1.0)$ & $4.9(0.9)$ & $4.5(0.9)$ \\
\hline Emotional burden $\ddagger$ & $4.6(0.8)$ & $4.8(0.5)$ & $4.0(1.0)$ & $4.9(0.4)$ & $4.8(0.6)$ \\
\hline
\end{tabular}

Table 3 Mean scores on process measures in the ACP Engagement Survey, by healthcare setting

\begin{tabular}{|c|c|c|c|c|c|}
\hline \multirow[b]{2}{*}{ Dimension } & \multicolumn{5}{|l|}{ Mean (SD)* } \\
\hline & Total $(\mathrm{N}=196)$ & Cancer $(n=49)$ & Hospital $(n=64)$ & Renal $(n=20)$ & Primary care $(n=63)$ \\
\hline Knowledge & $3.2(0.9)$ & $3.1(1.0)$ & $3.5(0.7)$ & $2.9(1.1)$ & $3.2(0.9)$ \\
\hline Contemplation & $2.3(0.8)$ & $2.3(1.0)$ & $2.4(0.7)$ & $2.3(0.6)$ & $2.2(0.7)$ \\
\hline Self-efficacy & $3.9(0.8)$ & $3.9(0.8)$ & $3.9(0.7)$ & $3.9(0.8)$ & $4.0(0.8)$ \\
\hline Readiness & $3.1(0.8)$ & $3.1(1.0)$ & $3.4(0.7)$ & $3.0(0.9)$ & $2.9(0.7)$ \\
\hline Total process measure score & $3.1(0.6)$ & $3.0(0.8)$ & $3.2(0.5)$ & $3.0(0.7)$ & $3.0(0.6)$ \\
\hline
\end{tabular}

shorter interview times than patients with cancer care $(\mathrm{p}=0.041)$, but not shorter than primary care patients $(\mathrm{p}=0.274)$.

\section{ACP process measures}

Mean process scales varied little across settings and ranged from 3.0 in cancer, renal and primary care settings to 3.2 in hospitalised patients. Scores were lowest for contemplation and highest for self-efficacy (table 3). Hospitalised patients reported the highest knowledge and readiness.

\section{ACP action measures}

The total mean action score was $11.2 \pm 5.6$, on a scale of 0-25. The lowest mean score was reported for patients in primary care settings $(9.6 \pm 4.9)$ and the highest was reported by hospitalised patients $(13.6 \pm 5.5)$ (table 4$)$.

Patients were least engaged in actions relating to flexibility (mean: $0.9 \pm 1.2$ ) and most engaged in actions relat-

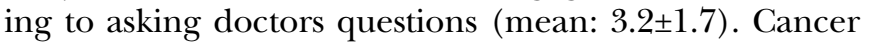
care setting patients reported the highest engagement in asking doctors questions (mean: 4.0 \pm 1.5 ). The mean action score related to decision makers was higher in hospital patients compared to all of the other settings.

\section{DISCUSSION}

In this multicentre study of 196 patients from hospital, cancer care, dialysis care and primary care settings, we found that the Survey was acceptable to patients and is feasible to administer in primary care, cancer care and dialysis care outpatient settings but less so in hospitalised patients.

Generally, mean ratings of acceptability, feasibility and clinical sensibility were in the good to very good range and lower in hospitalised patients (fair to good) than the other settings. Although less feasible and acceptable in hospitalised patients, the Survey compared favourably to another published survey of ACP engagement, assessed by the same measures in hospitalised patients, where most patients rated the survey as good, very good or excellent. ${ }^{10}$ In the current study, emotional burden was low in all settings, similar to a previous survey developed to assess the extent of ACP and satisfaction with communication and decision-making in seriously ill hospitalised adults, where patients rated the burden of participating in the interview as 2.8 on a $1-10$ scale of how burdensome. $^{10}$

Based on previous research surveying patients regarding ACP and end-of-life communication, the length of the current Survey may be problematic especially among sicker hospitalised patients. In the previous study of the 49-item Survey, the mean time to administer was 21.4 min (mean age of patients: 69 years; range: 55-92) in hospital inpatient and outpatient settings and community health clinics. ${ }^{9}$ In our study, the mean time to complete the 116-item version of the Survey ranged 
Table 4 Mean score on the action scales of the ACP Engagement Survey, by healthcare setting

\begin{tabular}{|c|c|c|c|c|c|}
\hline & \multicolumn{5}{|l|}{ Mean (SD) } \\
\hline & Total $(\mathrm{N}=196)$ & Cancer $(n=49)$ & Hospital $(n=64)$ & Renal $(n=20)$ & Primary care $(n=63)$ \\
\hline Total action score* $(n=196)$ & $11.2(5.6)$ & $10.1(5.5)$ & $13.6(5.5)$ & $11.1(6.2)$ & $9.6(4.9)$ \\
\hline DM subscale† $(n=196)$ & $2.9(1.5)$ & $2.2(1.6)$ & $3.7(1.2)$ & $2.7(1.5)$ & $2.8(1.2)$ \\
\hline QOL subscale $\neq(n=193)$ & $4.5(3.0)$ & $3.5(2.9)$ & $5.9(2.8)$ & $5.3(3.2)$ & $3.6(1.7)$ \\
\hline Health situations $†(n=193)$ & $2.4(1.5)$ & $1.5(1.5)$ & $3.0(1.4)$ & $2.3(1.7)$ & $2.0(1.4)$ \\
\hline Care at EOL† $(n=191)$ & $2.2(1.7)$ & $2.0(1.6)$ & $3.0(1.4)$ & $3.0(1.7)$ & $1.6(1.5)$ \\
\hline Flexibility subscale $(n=189)$ & $0.9(1.2)$ & $0.7(1.0)$ & $1.2(1.3)$ & $1.0(1.3)$ & $0.8(1.2)$ \\
\hline Ask questions subscale $(n=184)$ & $3.2(1.7)$ & $4.0(1.5)$ & $3.5(1.3)$ & $2.3(1.8)$ & $2.6(1.8)$ \\
\hline
\end{tabular}

from $34 \mathrm{~min}$ in cancer care settings to 1 hour in the hospital settings. The inclusion criteria of the hospitalised patients led to a sample $>10$ years older on average than the patients in the other settings. Hospitalised patients were likely experiencing an acute health event, which may explain the greater time to complete the survey. In previous studies on similar topics and in similar settings and populations that administered surveys taking on average 40-60 min, patients and family members reported that the duration was too long. ${ }^{10} 1213$ These findings have implications for administering research surveys in different settings and patient populations. For example, it may not be feasible or prudent to administer the Survey in the inpatient setting. Further revisions to the Survey are needed to address the length.

Engagement in ACP was low to moderate in this study. There were few process scales reaching or exceeding 4 of a possible 5 in any setting and the mean number of actions was fewer than half of the possible 25. In the study describing the earlier version of the Survey by Sudore et $a l,{ }^{9}$ mean process scores tended to be higher compared to our study. When used as an outcome measure in a pilot study of an ACP intervention among older adults, there was an increase in mean Likert process scores from 3.1 before the intervention to 3.7 one week after exposure to the intervention, and there were no significant changes in ACP actions. ${ }^{14}$ The lower scores found in the current study may be due to differences in the versions used with respect to the additional constructs (namely family and friends and medical wishes for end-of-life care) or due to additional burden of the added questions. Further studies may be required to determine benchmarks or thresholds for what is considered clinically meaningful ACP engagement.

Although our analysis of ACP engagement was exploratory, hospitalised patients appeared to be slightly more engaged in ACP than patients in other settings. Hospitalised patients reported the highest knowledge and readiness, and two to four more ACP actions on average, than patients from the other settings. We found that ACP engagement was similar across all outpatient settings, including cancer care and dialysis care, where we would expect greater illness severity and primary care settings where we would expect lesser illness severity. A previous study also found that illness severity, among outpatients, was not associated with stage of readiness for ACP. ${ }^{15}$

A strength of this study is the inclusion of wide-ranging healthcare settings across Canada. Since the legal details and health system approaches to ACP differ across the geographic areas where this study was conducted, the results may have greater generalisability to other settings than if the study were conducted in one province or city. There were also some limitations. First, the Survey asks the patient, not the surrogate, about the involvement of surrogates in ACP. This may result in social desirability bias as it is known that agreement between individuals and their surrogates on perceived extent of ACP involvement is often poor. ${ }^{16}$ Second, the Survey version used in this study contained new items that were added to the previous validated version based on feedback from stakeholders. Therefore, the version of the Survey used in this study may not have the same validity as the original version. Finally, the convenience sample is a limitation to the representativeness of patients in these settings, as sicker patients may not have been approached and respondents may have been more interested in ACP. If our sample included patients who were more interested in ACP and the results indicate low-to-moderate engagement in ACP, then true levels of ACP engagement may be even lower. In addition, patients were required to speak and understand English, not be cognitively impaired and hear well enough to participate in the interview. Most patients reported their ethnicity as Caucasian/white, which is not representative of major cities in Canada where the study was conducted; thus, there may be cultural differences that we cannot evaluate. ${ }^{17}$ Even though it was a convenience sample, our findings add important new knowledge about the feasibility, acceptability and clinical sensibility of the Survey.

\section{CONCLUSIONS}

Research on how to increase ACP in healthcare settings and its impact in those settings requires a measure of 
ACP engagement that links processes and actions. We have shown that the Survey demonstrated feasibility, acceptability and clinical sensibility in outpatient settings, but to a lesser extent among hospitalised patients. The main concern for feasibility was length. To facilitate the use of this measurement to evaluate interventions to improve engagement in ACP, further work is needed to reduce the length, especially if the Survey is to be used in hospitalised patients. ACP engagement was low to moderate across all settings, with older, hospitalised patients being the most engaged. Efforts to increase ACP engagement across these settings are warranted.

\author{
Author affiliations \\ ${ }^{1}$ Department of Family Medicine, McMaster University, Hamilton, Ontario, \\ Canada \\ ${ }^{2}$ Department of Clinical Epidemiology and Biostatistics, McMaster University, \\ Hamilton, Ontario, Canada \\ ${ }^{3}$ Clinical Evaluation Research Unit, Kingston General Hospital, Kingston, \\ Ontario, Canada \\ ${ }^{4}$ Department of Public Health, Queen's University, Kingston, Ontario, Canada \\ ${ }^{5}$ San Francisco Veterans Affairs Medical Center, San Francisco, California, \\ USA \\ ${ }^{6}$ Department of Medicine, Division of Geriatrics, University of California \\ San Francisco, San Francisco, California, USA \\ ${ }^{7}$ Covenant Health Palliative Institute, Edmonton, Alberta, Canada \\ ${ }^{8}$ School of Nursing, University of British Columbia, Kelowna, British \\ Columbia, Canada \\ ${ }^{9}$ Radiation Therapy Program, British Columbia Cancer Agency, Vancouver \\ Cancer Centre, Vancouver, Canada \\ ${ }^{10}$ Department of Medicine, McMaster University, Hamilton, Ontario, Canada
}

Collaborators Roy llan, Ingrid Brenner, Gillian Fyles, Marissa Slaven, Carol Mantle, Marilyn Swinton, Maureen Douglas.

Contributors AJB involved in analysis and interpretation of data, drafting of manuscript and critical revision. DE participated in acquisition of data, interpretation of data and critical revision. KF involved in study conception and design, acquisition of data, interpretation of data and critical revision. DKH involved in study conception and design, interpretation of data and critical revision. MH involved in study conception and design, acquisition of data, analysis and interpretation of data, drafting of manuscript and critical revision. MM involved in study conception and design, acquisition of data, interpretation of data and critical revision. CAR involved in study conception and design, acquisition of data, interpretation of data and critical revision. RS involved in study conception and design, interpretation of data and critical revision. JJY involved in study conception and design, acquisition of data, interpretation of data and critical revision. All authors approved the final submitted manuscript.

Funding This research received no specific grant from any funding agency in the public, commercial or not-for-profit sectors.

Competing interests None declared.

Ethics approval Hamilton Integrated Research Ethics Board, McMaster University.

Provenance and peer review Not commissioned; externally peer reviewed.
Data sharing statement Extra data can be accessed via the Dryad data repository at http://datadryad.org/ with the http://dx.doi.org/10.5061/dryad.1ng8c.

Open Access This is an Open Access article distributed in accordance with the Creative Commons Attribution Non Commercial (CC BY-NC 4.0) license, which permits others to distribute, remix, adapt, build upon this work noncommercially, and license their derivative works on different terms, provided the original work is properly cited and the use is non-commercial. See: http:// creativecommons.org/licenses/by-nc/4.0/

\section{REFERENCES}

1. Sinuff T, Dodek P, You JJ, et al. Improving end-of-life communication and decision making: the development of conceptual framework and quality indicators. J Pain Symptom Manage 2015;49:1070-80.

2. Silveira MJ, Kim SY, Langa KM. Advance directives and outcomes of surrogate decision making before death. $N$ Engl J Med 2010;362:1211-18.

3. Detering KM, Hancock AD, Reade MC, et al. The impact of advance care planning on end of life care in elderly patients: randomised controlled trial. BMJ 2010;340:c1345.

4. Heyland DK, Barwich D, Pichora D, et al. Failure to engage hospitalized elderly patients and their families in advance care planning. JAMA Intern Med 2013;173:778-87.

5. Teixeira AA, Hanvey L, Tayler C, et al. What do Canadians think of advanced care planning? Findings from an online opinion poll. BMJ Support Palliat Care 2015;5:40-7.

6. Ramsaroop SD, Reid MC, Adelman RD. Completing an advance directive in the primary care setting: what do we need for success? $J$ Am Geriatr Soc 2007;55:277-83.

7. Lewis $\mathrm{E}$, Cardona-Morrell M, Ong KY, et al. Evidence still insufficient that advance care documentation leads to engagement of healthcare professionals in end-of-life discussions: a systematic review. Palliat Med 2016. [Epub ahead of print 18 Jun 2016]. doi:10.1177/ 0269216316637239

8. Winter L, Parks SM, Diamond JJ. Ask a different question, get a different answer: why living wills are poor guides to care preferences at the end of life. J Palliat Med 2010;13:567-72.

9. Sudore RL, Stewart AL, Knight SJ, et al. Development and validation of a questionnaire to detect behavior change in multiple advance care planning behaviors. PLoS One 2013;8:e72465

10. Heyland DK, Pichora D, Dodek P, et al. The development and validation of a questionnaire to audit advance care planning. BMJ Support Palliat Care 2012;2:175-6.

11. Blackhall LJ, Murphy ST, Frank G, et al. Ethnicity and attitudes toward patient autonomy. JAMA 1995;274:820-5.

12. Heyland DK, Cook DJ, Rocker GM, et al. The development and validation of a novel questionnaire to measure patient and family satisfaction with end-of-life care: the Canadian Health Care Evaluation Project (CANHELP) Questionnaire. Palliat Med 2010;24:682-95.

13. Heyland DK, Jiang X, Day AG, et al, Canadian Researchers at the End of Life Network (CARENET). The development and validation of a shorter version of the Canadian Health Care Evaluation Project Questionnaire (CANHELP Lite): a novel tool to measure patient and family satisfaction with end-of-life care. J Pain Symptom Manage 2013;46:289-97.

14. Sudore RL, Knight SJ, McMahan RD, et al. A novel website to prepare diverse older adults for decision making and advance care planning: a pilot study. J Pain Symptom Manage 2014;47:674-86.

15. Fried TR, Redding CA, Robbins ML, et al. Stages of change for the component behaviors of advance care planning. J Am Geriatr Soc 2010;58:2329-36.

16. Fried TR, Redding CA, Robbins ML, et al. Agreement between older persons and their surrogate decision-makers regarding participation in advance care planning. J Am Geriatr Soc 2011;59:1105-9.

17. Canada S. Immigration and ethnocultural diversity in Canada. 2013. http://www12.statcan.gc.ca/nhs-enm/2011/as-sa/99-010-x/ 99-010-x2011001-eng.pdf (accessed 3 Jul 2015). 